\title{
Establishment of a bioluminescent imaging-based in vivo leukemia model by intra-bone marrow injection
}

\author{
MYOUNG WOO LEE ${ }^{1 *}$, HYE JIN KIM $^{1 *}$, KEON HEE YOO ${ }^{1}$, DAE SEONG KIM ${ }^{1}$, JIN MO YANG ${ }^{1}$, \\ HYE RYUNG KIM ${ }^{1}$, YOO HUN NOH ${ }^{1}$, HYUNJUNG BAEK ${ }^{2}$, HEECHUNG KWON ${ }^{2}$, MEONG HI SON ${ }^{1}$, \\ SOO HYUN LEE ${ }^{1}$, HEE WON CHEUH ${ }^{3}$, HYE LIM JUNG ${ }^{1}$, KI WOONG SUNG ${ }^{1}$ and HONG HOE KOO ${ }^{1}$ \\ ${ }^{1}$ Department of Pediatrics, Samsung Medical Center, Sungkyunkwan University School of Medicine; \\ ${ }^{2}$ Division of Radiation Oncology, Korea Institute of Radiological and Medical Sciences, Seoul; \\ ${ }^{3}$ Department of Pediatrics, Dong-A Medical Center, Dong-A University College of Medicine, Busan, Republic of Korea
}

Received May 22, 2012; Accepted July 23, 2012

DOI: 10.3892/ijo.2012.1634

\begin{abstract}
In vivo leukemia mouse models are usually generated by intraperitoneal (IP) or intravenous (IV) injection of leukemia cells. However, the pattern of leukemia development observed can be inconsistent. This study investigated injection directly into bone marrow [intra-bone marrow transplantation (IBMT)], the natural microenvironment of leukemia. A bioluminescent imaging-based leukemia animal model has been established by direct injection of a bioluminescent leukemia cells (CCRF-CEM/ fLuc) into NOD/SCID mouse tibia bone marrow and compared with models established by IP and IV routes. The comparison revealed that a bioluminescent in vivo leukemia model established via IBMT could recapitulate leukemia more faithfully and facilitate improved quantification of leukemia engraftment kinetics with a wider range of bioluminescent intensity than IP or IV. IBMT of bioluminescent leukemic cells allowed quantification of dose-dependent responses to anti-leukemic drugs, thus validating this model as a potential preclinical anti-leukemic drug screening system. IBMT-leukemia cells isolated from peripheral blood of the model mice and then injected into new recipients successfully established a second generation IBMT in vivo model and demonstrated the reproducibility of the model. Bioluminescent imaging-based analysis of this IBMT-leukemia model could provide a means for the comprehensive evaluation of treatment responses with enhanced sensitivity in preclinical studies.
\end{abstract}

Correspondence to: Professor K.H. Yoo or Professor H.H. Koo, Department of Pediatrics, Samsung Medical Center, 50 Irwon-dong, Gangnam-gu, Seoul 135-710, Republic of Korea

E-mail: hema2170@skku.edu

E-mail: hhkoo@skku.edu

${ }^{*}$ Contributed equally

Key words: leukemia, intra-bone marrow transplantation, firefly luciferase, bioluminescent imaging, animal model

\section{Introduction}

Recent advancements in molecular imaging have provided noninvasive analytical tools for continuous monitoring of disease progression and evaluating drug efficacy in preclinical models (1-3). The integration of a bioluminescent imaging system into preclinical leukemia model studies allows tracking of leukemic cell migration and dispersion patterns, and evaluation of therapeutic drug efficacy with enhanced sensitivity $(4,5)$. Many studies of acute lymphoblastic leukemia (ALL) have used in vivo leukemia models to explore new chemotherapeutic candidates and efficient treatment regimens in preclinical settings $(6,7)$.

The majority of in vivo leukemia models are initiated by intravenous (IV) or intraperitoneal (IP) injection of primary leukemia cells of patient origin or leukemia cell lines into immune-deficient mice such as NOD/SCID (8-10). Cells bearing a bioluminescent signal introduced in vivo have the potential to be tracked $(11,12)$. However, models that rely on IP or IV routes of introduction are likely to result in weak and/or delayed bioluminescent signals, which do not necessarily provide measurable parameters directly corresponding to progression of the disease. In particular, number and bioluminescent signals of leukemic cells within isolated organs and blood from IP and IV leukemia model mice are very low.

In this study, IP and IV models have been compared to intra-bone marrow transplantation (IBMT) leukemia animal model in which leukemic cells are introduced directly into their preferred microenvironment (13-15). IBMT is the only method that directly delivers leukemic cells to its preferred engraftment site with minimal loss of cells, thereby improving successful engraftment of leukemic cells for development of systemic disease model. IBMT model could be utilized to recapitulate human leukemia and allow consistent and sensitive bioluminescent evaluation.

\section{Materials and methods}

Cell culture. The T-cell acute lymphoblastic leukemia cell lines, Jurkat and CCRF-CEM and acute lymphocytic leukemia cell line, Reh were purchased from American Type Culture Collection (Manassas, VA) and cultured in RPMI-1640 (Life Technologies, 
Grand Island, NY) medium supplemented with $10 \%$ fetal bovine serum (FBS; Life Technologies), 1\% HEPES (Life Technologies), $100 \mathrm{U} / \mathrm{ml}$ of penicillin (Life Technologies), and $100 \mathrm{mg} / \mathrm{ml}$ of streptomycin (Life Technologies). These cell lines were maintained in a humidified $5 \% \mathrm{CO}_{2}$ atmosphere at $37^{\circ} \mathrm{C}$.

Introduction of f-luciferase gene via lentiviral infection. Reh, Jurkat and CCRF-CEM cell lines were transduced by addition of $1 \mathrm{ml}$ of viral stock consisting of pLenti6/V5-CMV viral vector (Life Technologies) encoding firefly luciferase. To facilitate the entrance of the viral vector into the cells, $8 \mu \mathrm{g} /$ $\mathrm{ml}$ of polybrene (Santa Cruz Biotechnologies, Santa Cruz, $\mathrm{CA})$ was added. Viable cells were washed with excess volume of phosphate-buffered saline (PBS; Biowest, Nuaille, France) 4 times and incubated directly in RPMI-1640 with $10 \%$ FBS, $1 \%$ penicillin/streptomycin, $1 \%$ HEPES and $5 \mu \mathrm{g} / \mathrm{ml}$ blasticidine (Sigma-Aldrich, St. Louis, MO) for clonal selection. To confirm successful transduction of f-luciferase gene, $1 \times 10^{6}$ clone cells were seeded in each well of a 6-well plate (Nalge Nunc, Naperville, IL) for bioluminescent imaging. Bioluminescent clones with the most luciferase activity of each cell lines were selected for injection and designated these bioluminescent leukemia cell lines as Reh/fLuc, Jurkat/fLuc and CCRF-CEM/ fLuc.

Bioluminescent leukemia animal model. NOD.CB17/scid Arc (NOD/SCID) mice were purchased from The Animal Resources (Canning Vale, WA, Australia) and maintained at the Laboratory Research Animal Center of the Samsung Biomedical Research Institute according to AAALAC approved protocols. Bioluminescent leukemia models were prepared by injecting 7 to 8 -week old NOD/SCID mice with $1 \times 10^{6}$ CCRF-CEM/fLuc via three different injection routes; intraperitoneal, intravenous (tail-vein) and intra-bone marrow (tibia) injection .

Bioluminescent imaging. These three types (IP, IV and IBMT) of leukemia in vivo models were compared through continuous bioluminescent monitoring using IVIS 100 imaging system (Xenogen Corporation, Alameda, CA). D-luciferin $(150 \mathrm{mg} / \mathrm{kg}$ ) (Xenogen) was injected intraperitoneally to each mouse prior to imaging. Mice were anesthetized with vaporized isofurane (Abbott Laboratory, Abbott Park, IL) and placed in imaging chamber. After 5.5 min, each animal was imaged alone in supine and prone positions with an exposure time of $1 \mathrm{~min}$ for each position weekly for 6 weeks. All bioluminescent image data were provided by Living Image software (version 1.0, Xenogen). Photons detected from leukemia models were converted to average radiance (photon $/ \mathrm{sec} / \mathrm{cm}^{2} / \mathrm{sr}$ ). Average radiance values are quantitative data obtained from region of intensity (ROI) where photons emitted by bioluminescent cells of assigned rectangular area over the whole body of each mouse. Both luminescence and image data were analyzed using Living Image software.

Flow cytometric analysis. To validate bioluminescent correlation with peripheral leukemic cells of leukemia burden in vivo model, mice were sacrificed at week 6 after bioluminescent images were obtained. Peripheral blood (PB), bone marrow (BM) aspirates, and spleen were obtained to isolate human leukocytes using Ficoll-Paque ${ }^{\mathrm{TM}}$ Plus (GE Healthcare, Uppsala, Sweden) solution. Residual red blood cells were removed using erythrocyte lysis buffer (Qiagen, Hilden, Germany) prior to double staining the isolated cells with phycoerythrin (PE)-conjugated anti-human CD45 (hCD45) (BD Pharmigen ${ }^{\mathrm{TM}}$, San Diego, CA) and fluorescein isothiocyanate (FITC)-conjugated anti-mouse CD45 (mCD45) (BD Pharmigen ${ }^{\mathrm{TM}}$ ) antibodies. PB and BM of the mice treated with vincristine and methylprednisolone were also analyzed to determine the correlation between bioluminescent changes in responses to vincristine or methylprednisolone within leukemia burdened mice. Mononuclear cells isolated from PB of the second generation IBMT leukemia model was evaluated with hCD45 and mCD45 antibodies once again to validate that human leukemic cell of the first IBMT model were responsible for the development of bioluminescent leukemia model.

Cell viability assay: Alamarblue assay. In vitro sensitivity of CCRF-CEM/fLuc cell line to vincristine and methylprednisolone was validated using Alamarblue ${ }^{\circledR}$ (Life Technologies) assay and luminescence assay prior to transplantation. CCRF-CEM/ fLuc cells $\left(1 \times 10^{5}\right)$ were seed in each well of 96-well. Five columns of 4 wells were treated with vincristine at concentrations of $0.1,0.5,1,5$, and $10 \mathrm{ng} / \mathrm{ml}$. Cells were exposed to vincristine for $48 \mathrm{~h}$ before endpoint data analysis were conducted using Alamarblue assay. Replicate vincristine in vitro assay was done and analyzed with bioluminescent imaging, $2 \mu \mathrm{l}$ $(300 \mu \mathrm{g} / \mathrm{ml})$ of D-luciferin was added to each well, and image of the in vitro assay plate was obtained. Another 96-well plate seeded with CCRF-CEM/fLuc cells of the identical experimental setting as vincristine was prepared to determine effective cytotoxic concentration of methylprednisolone, 100, 250, 500 and $1000 \mu \mathrm{g} / \mathrm{ml}$ of methylprednisolone were treated for $48 \mathrm{~h}$. Alamarblue and bioluminescent assay were also used to assess in vitro responses of CCRF-CEM/fLuc to methylprednisolone.

In vivo evaluation of anti-leukemic drug responses. Mice in groups of three were treated with two different vincristine (Hospira, Mulgrave, Australia) concentrations starting at week 3 . Vincristine $(0.1$ and $0.5 \mathrm{mg} / \mathrm{kg})$ in $100 \mu \mathrm{l}$ PBS were injected intravenously into tail vein 3 times in 7 days interval. Control mice were injected with $100 \mu \mathrm{l}$ PBS every week as well. The effects of vincristine on leukemia mouse models were monitored once a week from the initial treatment for 21 days. Prior to administration of vincristine, bioluminescent images of the mice were obtained. Standard bioluminescent imaging protocol was used to monitor changes in vincristine treated mice. Another set of IBMT leukemia mouse model was prepared according to methods described previously. These mice were treated weekly with two different concentrations of methylprednisolone (Pfizer, Puurs, Belgium) starting at week 3. Methylprednisolone (2 and $10 \mathrm{mg} / \mathrm{kg}$ ) in $100 \mu \mathrm{l}$ PBS were administered intravenously into tail vein. Monitoring responses to methylprednisolone was done by weekly imaging and images were obtained according to the protocol described in bioluminescent imaging section of Materials and methods.

Secondary engraftment of CCRF-CEM/fLuc cells. CCRF-CEM/ fLuc cells were isolated from peripheral blood of IBMTleukemia mouse model via density gradient removal of RBC using Ficoll solution. Cells were maintained in RPMI-1640 
with $10 \%$ FBS, $1 \%$ antimycotic-antibiotic and $1 \%$ HEPES. To assess luciferase expression level of ex vivo CCRF-CEM/fLuc, the number of cells ranging between $10^{3}$ and $10^{7}$ cells were suspended in $100 \mu \mathrm{l}$ of PBS and plated on flat-bottom 96-well plate. $2 \mu \mathrm{l}(300 \mu \mathrm{g} / \mathrm{ml})$ of D-luciferin was added to each well, then bioluminescent signal were detected for $30 \mathrm{sec}$ with CCD camera. Bioluminescent leukemia mouse model was reconstituted by injecting $10 \mu \mathrm{l}$ of $1 \times 10^{6}$ of the isolated CCRF-CEM/ fLuc cells directly into tibia of the secondary recipient mice $(n=5)$. Leukemia development was monitored weekly. Mice were given an intraperitoneal injection of D-luciferin $(150 \mathrm{mg} /$ $\mathrm{ml}$ ) and imaged $5.5 \mathrm{~min}$ later. Each mouse in prone and supine positions according to previously described in bioluminescent imaging section of Materials and methods.

Statistical analysis. Statistical analysis of the data was performed using Prism 5 (Graphpad Software, La Jolla, CA). In vitro data represent mean of triplicates and correlation between bioluminescent signals and number of viable leukemic cells was determined via Spearman correlation analysis. Kruskal-Wallis analysis was performed with Dunn Multiple Comparison test to compare experimental groups to controls.

\section{Results}

Establishment of bioluminescent leukemia cell lines stably expressing firefly luciferase. Bioluminescent leukemia cell lines were established using a firefly luciferase (fLuc) gene-encoding vector delivered via lentiviral infection into human ALL cell lines Reh, Jurkat and CCRF-CEM. Stable expression of fLuc in these cell lines was confirmed by analyzing the bioluminescent intensity of $10^{6}$ cells of each, quantified in average radiance (photon $/ \mathrm{sec} / \mathrm{cm}^{2} / \mathrm{sr}$ ) (Fig. 1A). The bioluminescent intensity was measured in serial dilutions $\left(10^{7}-10^{3}\right)$ of CCRF-CEM/fLuc cells (Fig. 1B), and the signal intensity was converted into average radiance (photon $/ \mathrm{sec} / \mathrm{cm}^{2} / \mathrm{sr}$ ) (Fig. 1C). Bioluminescent intensity was directly proportional to the number of viable cells $(\mathrm{p}<0.001)$. To assess purity of the established human cell lines, CCRF-CEM and CCRF-CEM/fLuc cells were analyzed by flow cytometry for expression of common human leukocyte antigen marker, CD45 using PE-conjugated hCD45 and FITC-conjugated mCD45 antibodies (Fig. 1D). Double staining analysis ensures that only human-origin cell lines were used for leukemia model development. Both cell lines were positive for hCD45 and negative for $\mathrm{mCD} 45$. These results established the correlation between viable, luciferase-expressing CCRF-CEM/fLuc cells and bioluminescent signal intensity.

In vivo monitoring of bioluminescent leukemia progression in a NOD/SCID mouse model. NOD/SCID mice were inoculated with $10^{6} \mathrm{CCRF}-\mathrm{CEM} / \mathrm{fLuc}$ cells via IP, IV or IBMT routes (Fig. 2A) and examined using the bioluminescent imaging system every week for 6 weeks. For mice injected with IP, bioluminescent signals were localized and detected only at the site of injection (Fig. 2B); however, in mice inoculated with IV, the average radiance from luciferase activity of viable CCRF-CEM/fLuc was within the detectable range but was insufficient for visualization during the first three weeks after transplantation. Bioluminescent signals from the lower border of the sternum on the ventral side, head and upper sternum on the
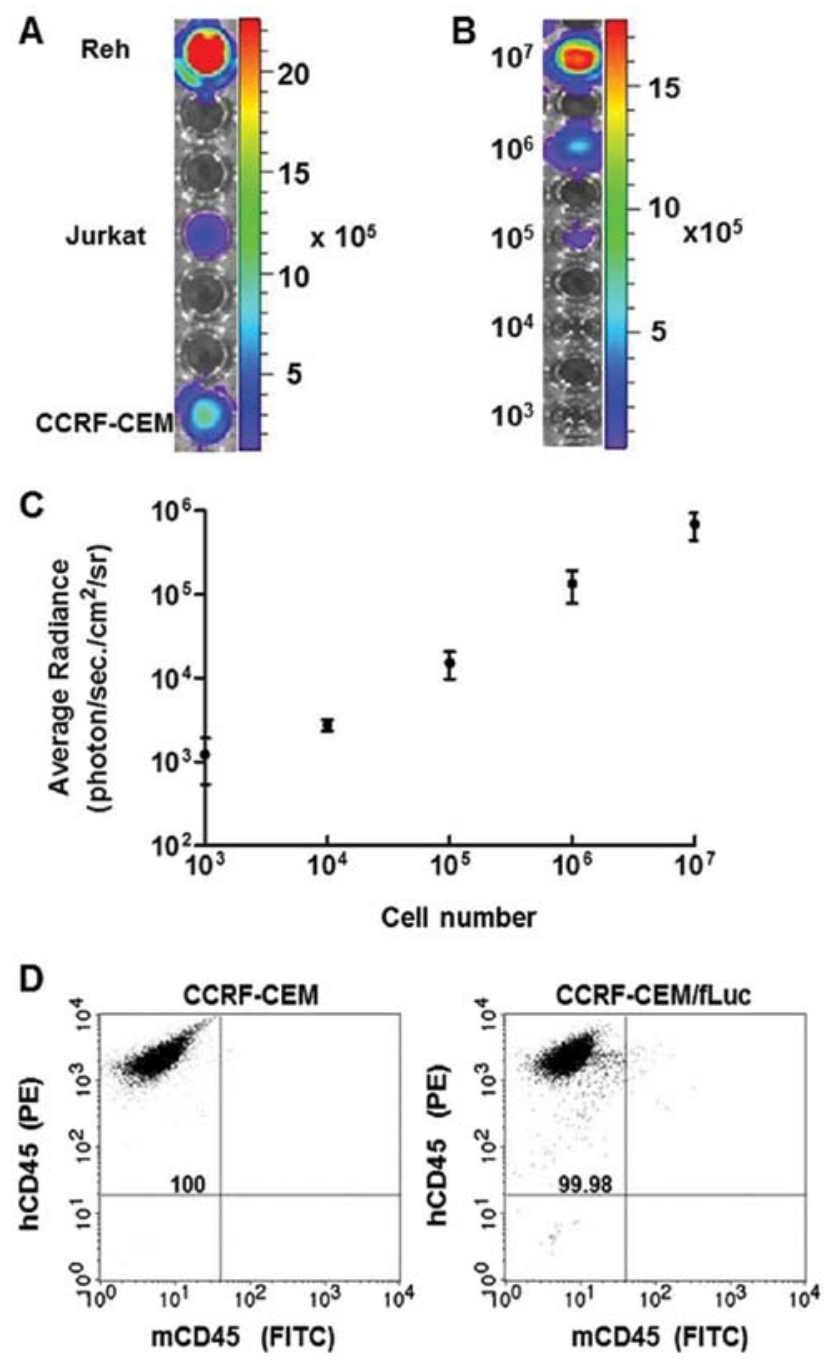

Figure 1. Establishment of human leukemia cell lines stably expressing firefly luciferase. (A), Bioluminescence of Reh, Jurkat and CCRF-CEM cell lines stably expressing fLuc. After clonal selection with blasticidine, $10^{6}$ cells from each cell line were evaluated for fLuc activity. (B), CCRF-CEM/fLuc was serially diluted from $10^{7}$ to $10^{3}$ cells (five concentrations in all) and then imaged after addition of D-luciferin to each diluted cell suspension. (C), Correlation between the numbers of viable CCRF-CEM/fLuc cells and its mean bioluminescent intensity (photon $/ \mathrm{sec} / \mathrm{cm}^{2} / \mathrm{sr}$ ) depicted by plotting the number of cells against average radiance values of serially diluted cells $(\mathrm{p}<0.001)$. (D), Flow cytometric profiles of CCRF-CEM and CCRF-CEM/fLuc (transduced by lentiviral vector encoding luciferase). Both cell lines expressed hCD45 confirming the purity of the cell line.

dorsal side became visible at week 4 (Fig. 2C). Unlike leukemia models prepared via IP and IV injections, bioluminescent signals of mice inoculated via IBMT were detectable in the tibia as early as one week after the inoculation of CCRF-CEM/fLuc cells. At week 4 , strong bioluminescent signals were detected in mice in both supine and prone positions. The highest intensity signals were mainly detected in the head and backbone in the prone position, and in the upper sternum and hind legs in the supine position (Fig. 2D). Fig. 2E shows the mean radiance values for each inoculation group $(n=15)$ over 6 weeks of monitored time. At week 6, IBMT model exhibited significantly stronger bioluminescent signals $(\mathrm{p}<0.001)$ than IP or IV mediated leukemia models. These results indicate that the visualization and quantification of leukemia progression can vary with the leukemia cell transplantation method. 
A
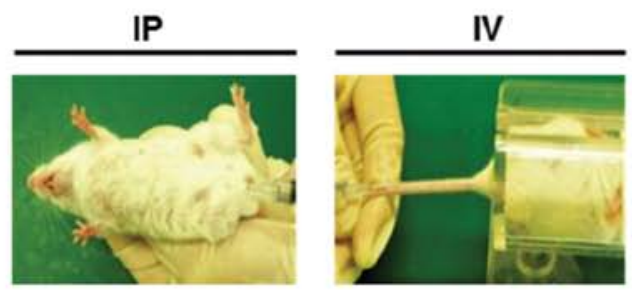

IBMT

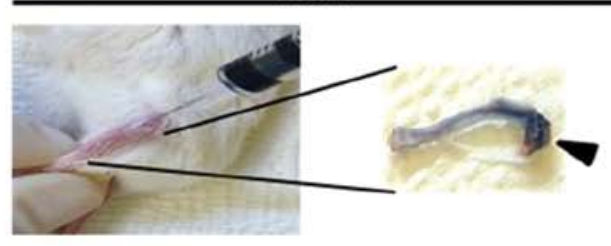

E

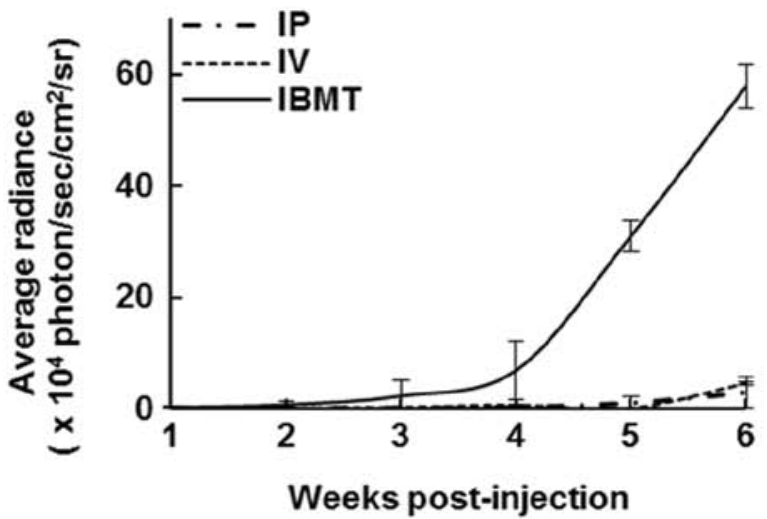

B

IP
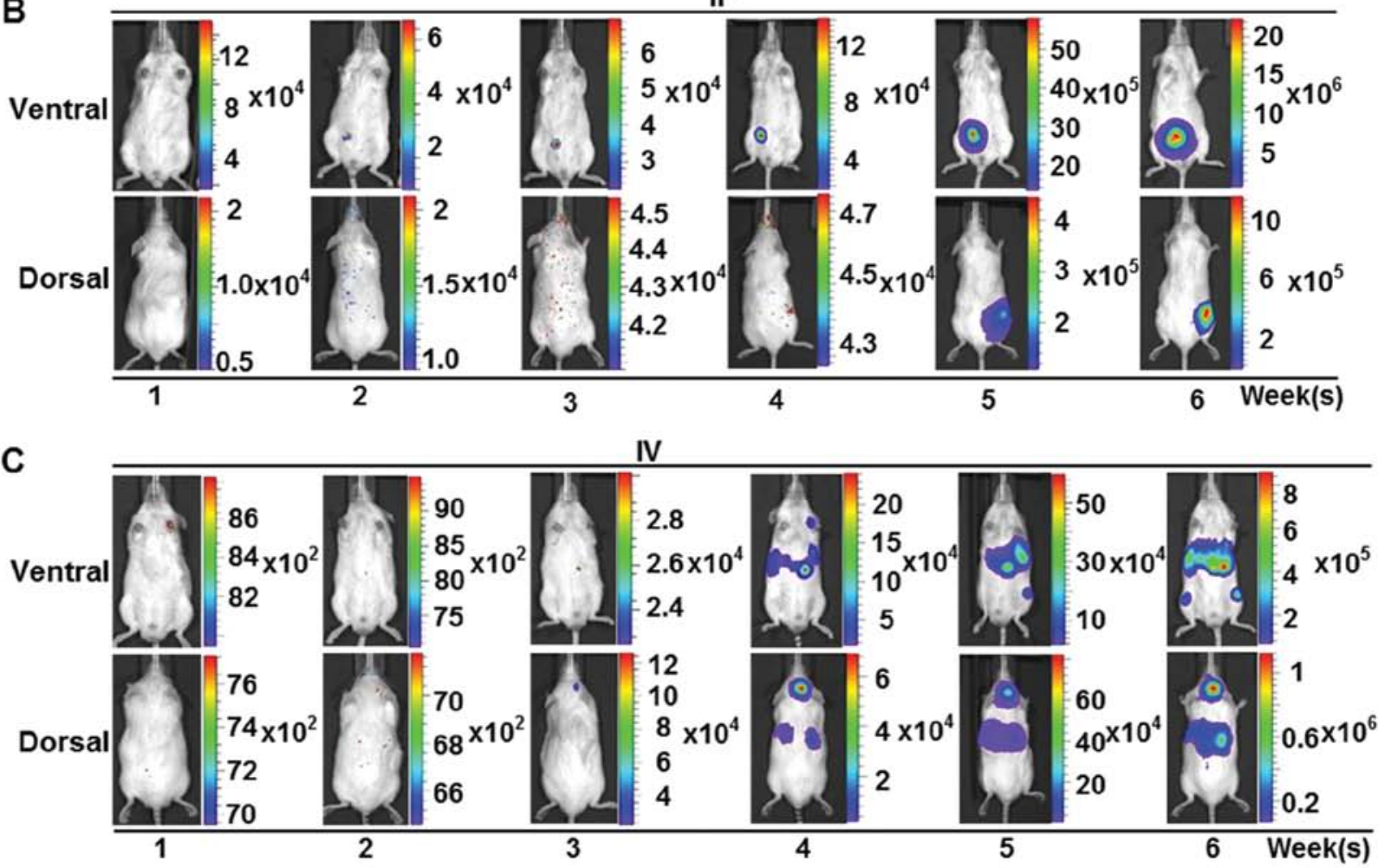

D

IBMT

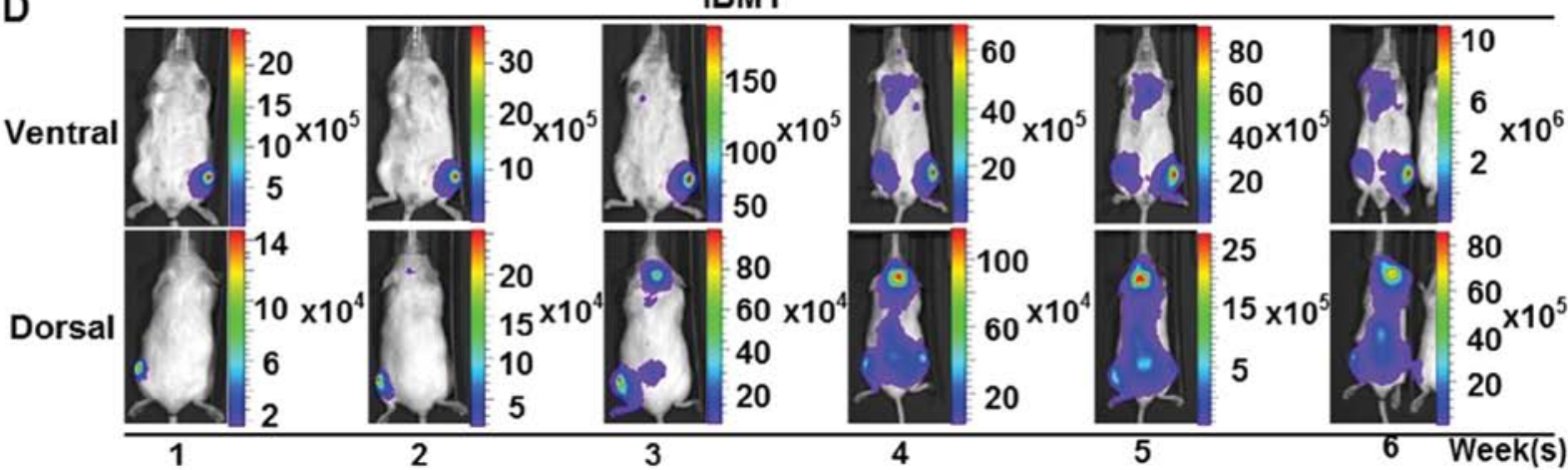

Figure 2. Comparative bioluminescent imaging analysis of bioluminescent in vivo leukemia models established using three injection methods: IP, IV and IBMT. (A), Demonstrations of the three injection methods used to xenograft bioluminescent leukemic cells in 7-week old NOD/SCID mice. Trypan blue dye was used to demonstrate IBMT. Successful IBMT was confirmed in the dissected tibia by the presence of trypan blue dye in the bone marrow cavity (indicated by arrow). (B-D), Bioluminescent intensity maps of representative mice from IP, IV and IBMT leukemia model groups monitored from week 1 to 6 post-injection of 10 CCRF-CEM/fLuc cells. The signal intensity scale is shown in color as a representation of the optimized bioluminescent signals range of luciferase-expressing leukemic cells. It includes normalized bioluminescent light intensity range: highest intensity (red) and lowest intensity (blue). The representative mice show differing dispersal patterns and proliferation rates of leukemic cells. (E), Graph comparing mean bioluminescent signals obtained from IP ( $\mathrm{n}=15$ ), IV ( $\mathrm{n}=15$ ) and IBMT $(\mathrm{n}=15)$ leukemia model groups over 6 weeks using the optical imaging system, IVIS $100(\mathrm{p}<0.001)$. 
A
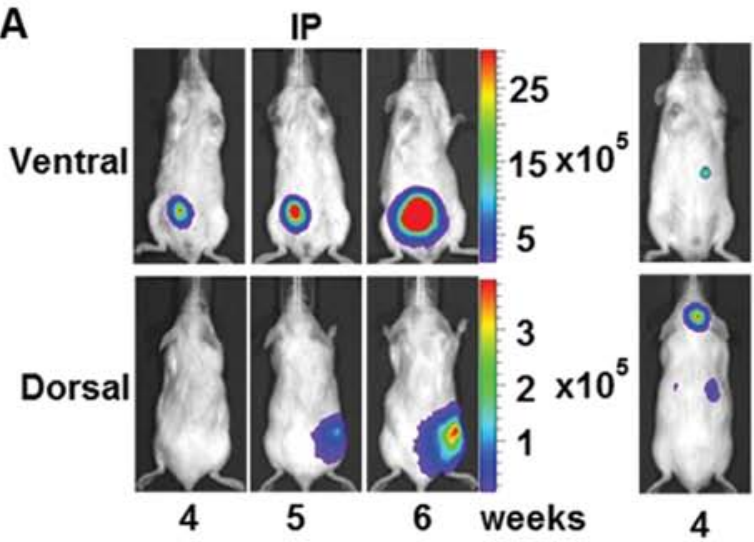

IV
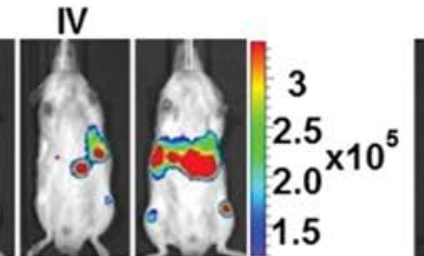

IBMT

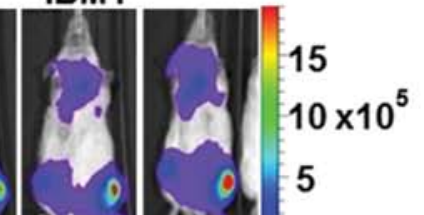

B

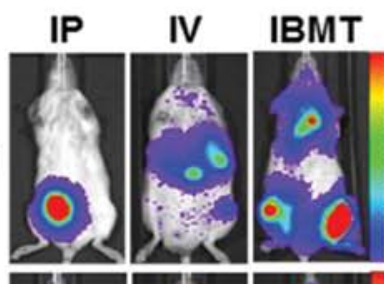

Ventral
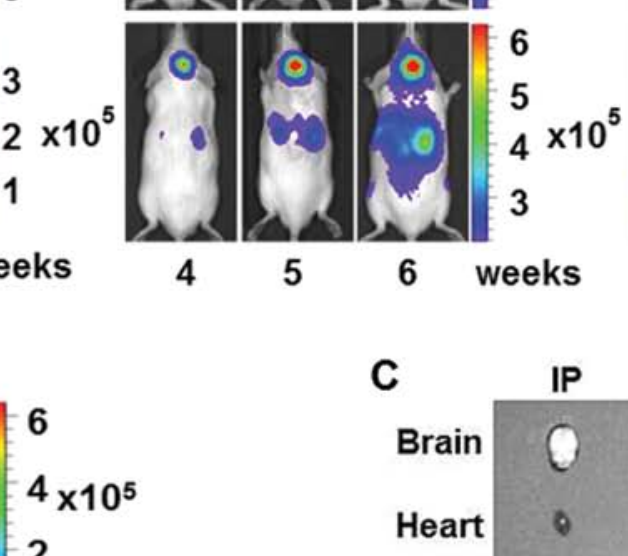

6

weeks

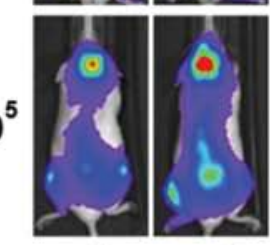

4

5

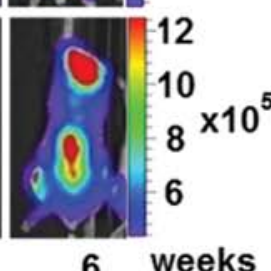

6 weeks

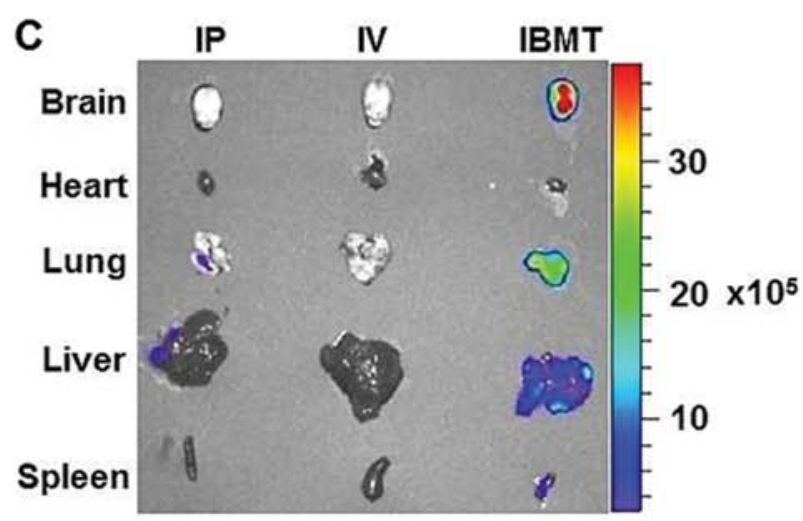

D
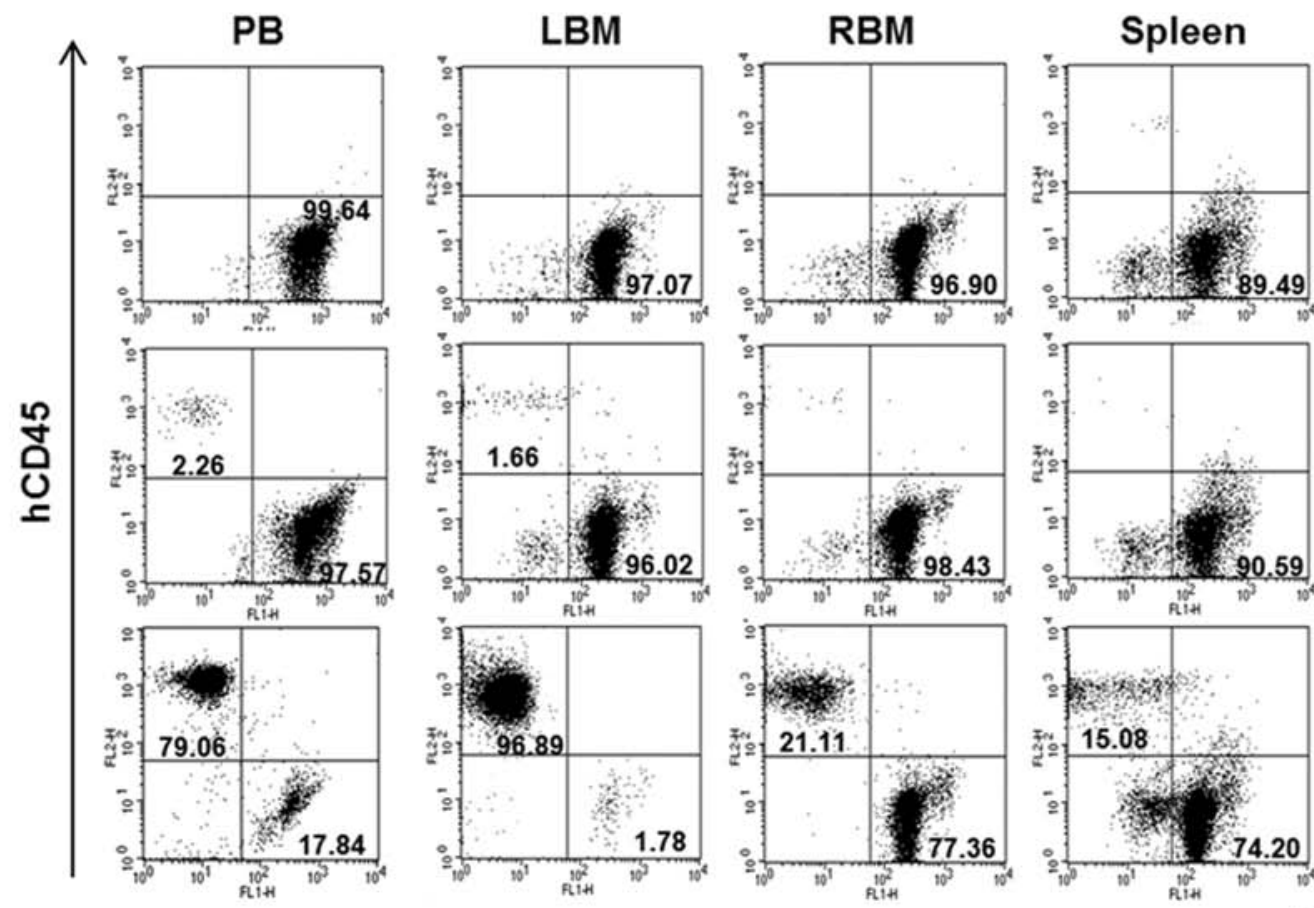

IP
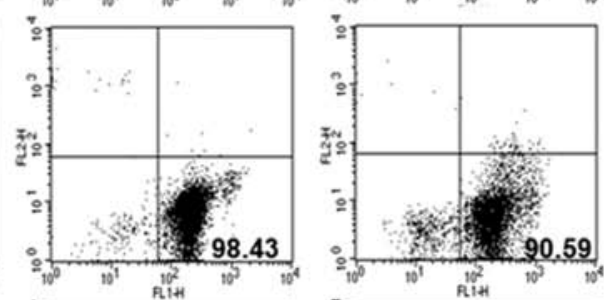

IV
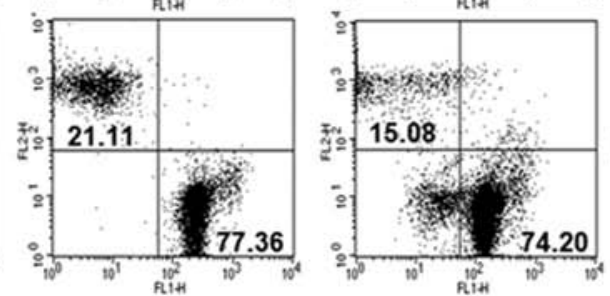

IBMT

mCD45

Figure 3. Quantitative correlation between leukemia burden and level of organ infiltration by leukemic cells established with the bioluminescent IBMT model. (A), The bioluminescent intensity of each group from week 4 to 6 was normalized to show the significant bioluminescent signal range within each group using a signal intensity scale. Changes of bioluminescent intensity over the course of monitoring are shown for clearer comparative analysis within each group. (B), Representative images from each group at week 5 with a normalized signal intensity scale, which includes the bioluminescent signal range from IP, IV and IBMT model mice, for comparative analysis. (C), Bioluminescent images of organs from representative mice from each group isolated at week 5 . The only bioluminescent signals detected were in the IBMT model mouse brain, lung, liver and spleen. (D), The level of human leukemic cells in IP, IV and IBMT models was analyzed using flow cytometric analysis of PB, spleen and BM from left (LBM) and right tibia (RBM). The human leukemic cell burden of the IBMT model was significantly higher in the peripheral blood $\left(79.06 \%\right.$ of hCD $45^{+}, 17.84 \%$ of $\left.\mathrm{mCD} 45^{+}\right)$, BM $\left(96.89 \%\right.$ of hCD $45^{+}$in left tibia and $21.11 \%$ of hCD $45^{+}$in right tibia in the prone position), and spleen $\left(15.08 \%\right.$ of hCD $45^{+}, 74.20 \%$ of $\left.\mathrm{mCD} 45^{+}\right)$of IBMT mice than in mice injected IP and IV. 
Comparative analysis of the route of transplantation for establishing a bioluminescent leukemia in vivo model. To compare leukemia burden level in IP, IV and IBMT leukemia models with accuracy, calibrated signal intensity ranges representing the optimal range of signals above noise but below saturation level detected by CCD camera were assigned to each type of leukemia mouse model. In particular, images of week 4, 5 and 6 were compiled and compared with calibrated signal intensity ranges within each type leukemia model (Fig. 3A). The gradual increase in bioluminescent signal intensity from week 4 through 6 images was observed. In addition, images from one representative mouse at week 5 in each of the IP, IV and IBMT groups were pooled and compared within the calibrated signal intensity range (Fig. 3B). The IBMT leukemia model exhibited the strongest signal intensity; this represents the highest degree of leukemia burden among the three leukemia models. The corresponding bioluminescent intensity of isolated organs (brain, heart, lung, liver and spleen) from these leukemia model mice at week 5 was assessed (Fig. 3C). Organ images revealed that only those from the IBMT leukemia model had strong bioluminescent signals. It was investigated by flow cytometry whether the bioluminescent areas observed corresponded to leukemic cells in the organs from the IBMT leukemia model. Disease burden, i.e., human leukemic cells, in PB, BM and spleen cells from IP, IV and IBMT leukemia model mice sacrificed at week 5 post-injection was quantified by flow cytometric analysis of hCD45 and mCD45 expression (Fig. 3D). $\mathrm{BM}$ aspirates from the tibia (LBM) of IBMT mice contained mainly hCD $45^{+}$cells $(96.89 \%)$, PB contained $79.06 \%$ hCD $45^{+}$ cells and the spleen contained $15.08 \%$ hCD $45^{+}$cells. However, in the IP model, less than $1 \%$ of cells in PB, BM and spleen were $\mathrm{hCD} 45^{+}$, and in the IV model, less than $2.26 \%$ of cells in $\mathrm{PB}, \mathrm{BM}$ and spleen were $\mathrm{hCD} 45^{+}$. Overall, results from comparative analyses of IP, IV and IBMT leukemia models indicate that detectable bioluminescent leukemia development in NOD/SCID can be effectively achieved using an IBMT route of administration.

Bioluminescent IBMT leukemia model enables non-invasive monitoring of anti-leukemic treatment responses with greater sensitivity. To demonstrate that the IBMT bioluminescent leukemia model can be used to show the correlation between in vivo responses to anti-leukemic treatments and its corresponding bioluminescent intensity changes, IBMT leukemia mice were monitored by whole body bioimaging after treatment with anti-cancer drugs, vincristine and methylprednisolone. Response to the drugs was first tested in vitro. Viability of the leukemia cell line decreased as the concentration of vincristine increased (Fig. 4A). Bioluminescent evaluation of the viability assay confirmed a decrease in bioluminescent viable leukemic cells with an increase of vincristine concentration (Fig. 4B and C). Next, bioluminescent imaging of the IBMT leukemia model was evaluated during treatment with vincristine at two concentrations; $0.1,0.5 \mathrm{mg} / \mathrm{kg}$ and PBS (Fig. 4D). Where bioluminescence at week 3 was initially detected pre-treatment, the intensity reduced significantly $(\mathrm{p}<0.01)$ after three weekly doses of vincristine at $0.5 \mathrm{mg} / \mathrm{kg}$ (Fig. 4E). The control group mouse (no vincristine) became moribund shortly after the image was taken at week 6 . The reduction of whole body bioluminescent signals observed with the higher dose of vincristine was also observed in isolated organ images. The higher concentration of vincristine resulted in a reduction in leukemic cell infiltration of organs (Fig. 4F). The reduction in bioluminescent signals from vincristine-treated mice was validated by flow cytometric evaluation of isolated PB and BM cells (Fig. 4G). The percentage of $\mathrm{hCD} 45^{+}$cells in $\mathrm{PB}$ of 0.1 and $0.5 \mathrm{mg} / \mathrm{kg}$ vincristine-treated mice was lower than in PB from a control mouse. BM aspirates taken at the site of injection (left tibia) of the control IBMT mice and $0.1 \mathrm{mg} / \mathrm{kg}$ vincristine-treated IBMT mice were greater than $90 \% \mathrm{hCD}^{+} 5^{+}$. In contrast, analysis of BM aspirates from the injection site of a $0.5 \mathrm{mg} / \mathrm{kg}$ vincristine-treated mouse revealed a significantly lower percentage of hCD $45^{+}$ cells $(25.66 \%)$. In BM aspirates from the right tibia, compared to the control (92.23\%), the percentage of $\mathrm{hCD}^{2} 5^{+}$cells in $0.1 \mathrm{mg} / \mathrm{kg}(10.79 \%)$ and $0.5 \mathrm{mg} / \mathrm{kg}(0.58 \%)$ vincristine-treated mice was significantly lower. In addition, a viability assay of CCRF-CEM/fLuc with methylprednisolone was conducted. The endpoint analyses from Alamarblue assay (Fig. 5A) and bioluminescent evaluation (Fig. 5B and C) showed that the viability of the leukemic cells decreased with an increase in methylprednisolone concentration. The mice were treated with PBS (control, $\mathrm{n}=9), 2 \mathrm{mg} / \mathrm{kg}(\mathrm{n}=9)$ or $10 \mathrm{mg} / \mathrm{kg}(\mathrm{n}=9)$ methylprednisolone once a week for 3 weeks. Each dotted line represents average bioluminescent intensity values for each treatment group (Fig. 5D). Methylprednisolone treatments $(10 \mathrm{mg} / \mathrm{kg})$ resulted in significantly $(\mathrm{p}<0.01)$ lower bioluminescence than $2 \mathrm{mg} / \mathrm{kg}$ or PBS treatment groups at week 6 . Before (week 3) and after (week 6) images of representative mice treated with methylprednisolone show that the IBMT leukemia model can capture sensitive treatment responses (Fig. 5E). The results indicate that bioluminescent images of the IBMT in vivo leukemia model can provide an accurate representation of sensitive responses to candidate drugs.

IBMT facilitates the establishment of a bioluminescent leukemia in vivo model in secondary recipient mice. To demonstrate that the IBMT route of administration is an effective method for generating a reproducible in vivo leukemia model, leukemia cells from $\mathrm{PB}$ of the first generation leukemia model mice at week 6 (Fig. 6A) were isolated. PB mononuclear cells from an IBMT leukemia model mouse that comprised $71.83 \% \mathrm{hCD}^{+} 5^{+}$ cells along with $25.63 \% \mathrm{mCD}^{+} 5^{+}$cells (Fig. 6B). The luciferase expression level of the ex vivo leukemic cells was evaluated to ensure that these cells maintained characteristics of the human leukemia cell line, CCRF-CEM/fLuc, which was originally transplanted. Bioluminescent images of $10^{5}, 10^{6}$ and $10^{7}$ isolated, ex vivo expanded cells were obtained and their average radiance values were plotted (Fig. 6C and D). These ex vivo cells proliferated and stably expressed luciferase during long-term in vitro culture (data not shown). After ex vivo expansion, the isolated leukemic cells were xenografted into the left tibia of secondary recipient NOD/SCID mice (Fig. 6E). The second generation model mice showed a similar course of bioluminescent intensity to that of the first (Fig. 6F). Bioluminescent activity of human leukemic cells in PB from the second generation was quantified by flow cytometric analysis 6 weeks after transplantation of the first generation leukemic cells. $\mathrm{PB}$ comprised $64.36 \% \mathrm{hCD}^{+} 5^{+}$ cells and $24.70 \% \mathrm{mCD} 45^{+}$cells (Fig. 6G). These results indicate that the IBMT route is able to generate a reproducible in vivo leukemia model. 
A

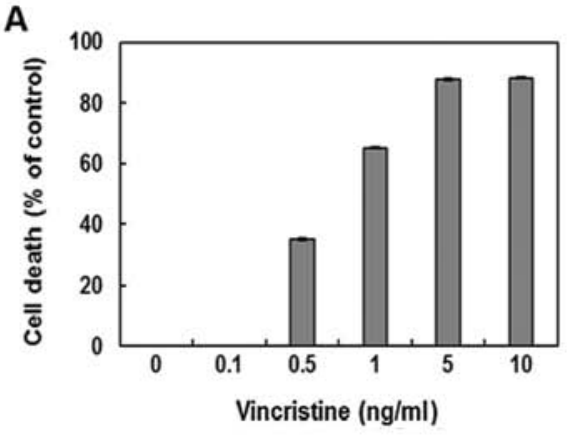

D

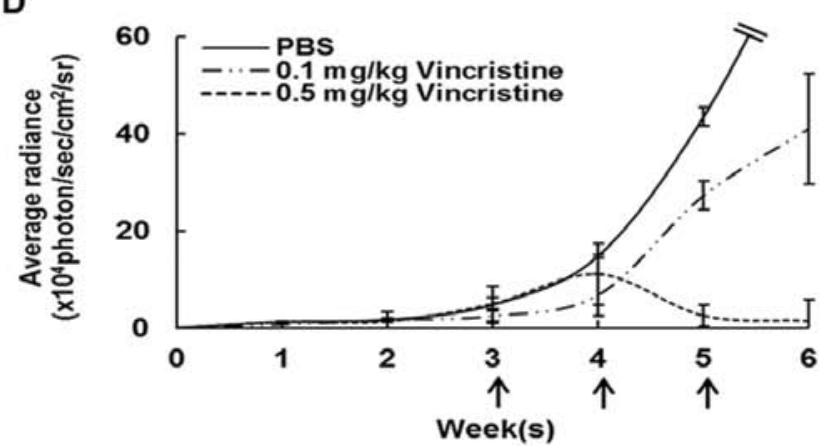

B

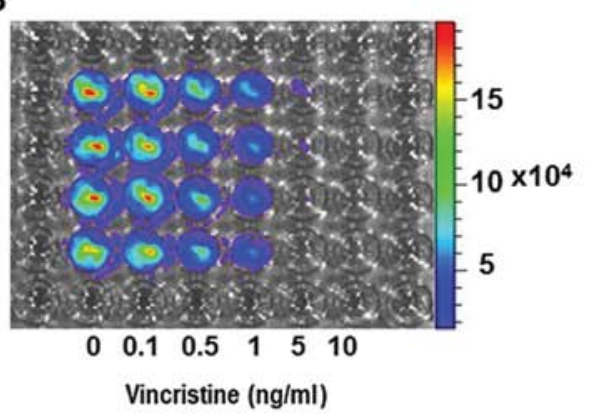

C

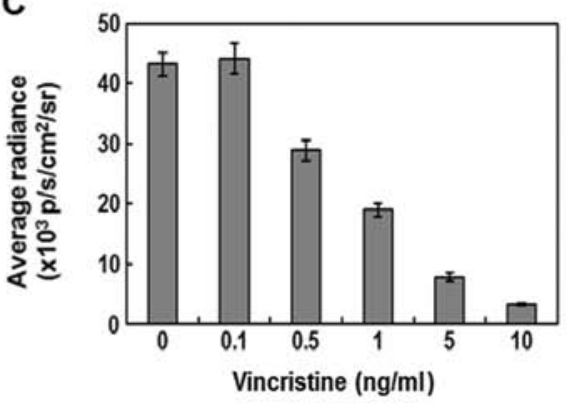

F

E

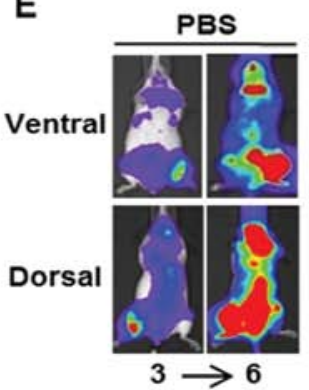

Vincristine Vincristine

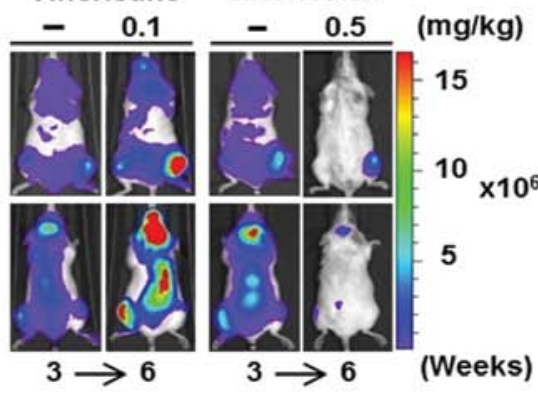

G

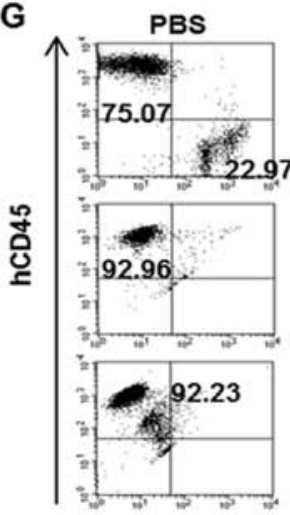

$0.1 \mathrm{mg} / \mathrm{kg}$

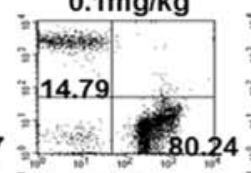

$0.5 \mathrm{mg} / \mathrm{kg}$
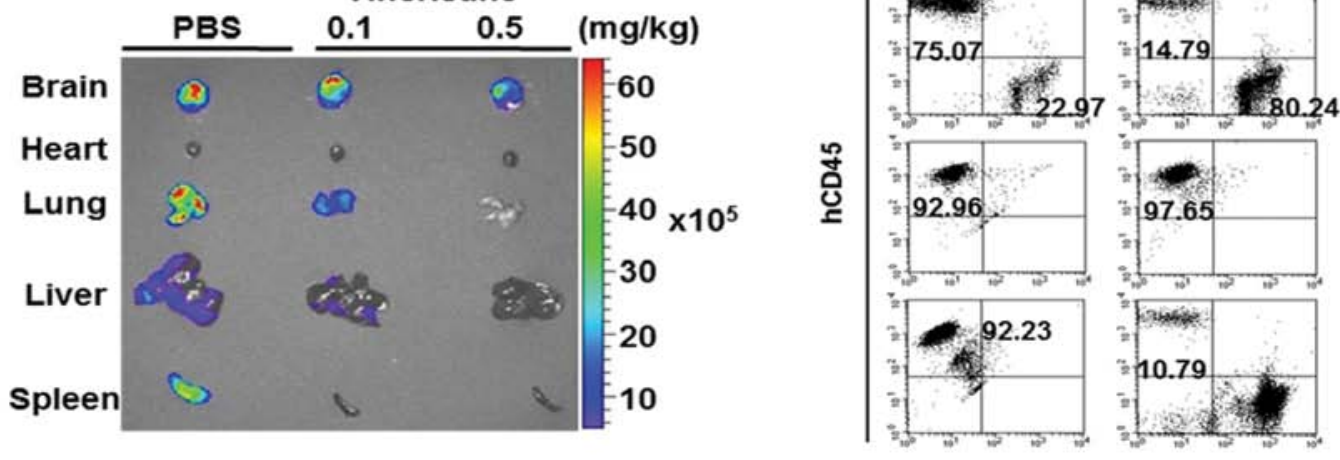

mCD45

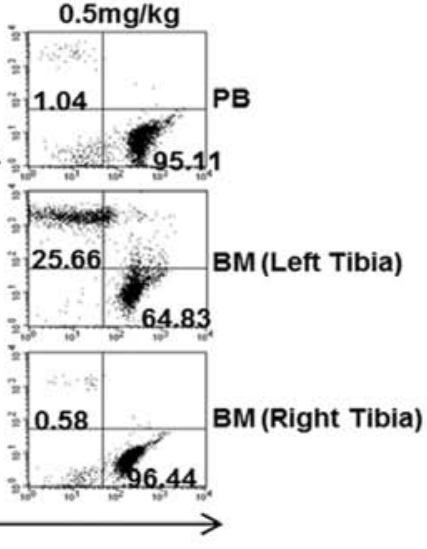

Figure 4. Bioluminescent imaging-based evaluation of sensitivity to vincristine in the IBMT model. (A), In vitro sensitivity of CCRF-CEM/fLuc to vincristine $(0,0.1,0.5,1,5$, or $10 \mathrm{ng} / \mathrm{ml})$ was determined using Alamarblue assay. $(\mathrm{B}, \mathrm{C})$, Bioluminescence of the cell line treated with vincristine at different concentrations. Cell death of CCRF-CEM/fLuc cell line increased and bioluminescence decreased with vincristine concentration. (D), In vivo sensitivity of bioluminescent leukemic cell-xenografted mice to 0.1 and $0.5 \mathrm{mg} / \mathrm{kg}$ vincristine. Weekly treatment responses were evaluated with bioluminescent imaging. Quantification of bioluminescent CCRF-CEM/fLuc responses over time to weekly intravenous tail-vein treatment of vincristine $0.1 \mathrm{mg} / \mathrm{kg}(\mathrm{n}=9), 0.5 \mathrm{mg} / \mathrm{kg}(\mathrm{n}=9)$ or PBS $(\mathrm{n}=9)$ for 3 weeks. (E), Bioluminescent leukemia responses in control mouse and to 0.1 and $0.5 \mathrm{mg} / \mathrm{kg}$ vincristine were evaluated by comparing images before treatment at week 3 and after final treatment at week $6(\mathrm{p}<0.01)$. (F), Bioluminescent organ images of control mouse and mice treated with high or low concentrations of vincristine taken 1 week after final treatment. (G), Flow cytometric analyses of leukemic cell burden in PB and BM of vincristine-treated mice and six control mice.

\section{Discussion}

The comparison of in vivo leukemia models established with three different injection methods in this study revealed that IBMT of leukemic cells with bioluminescence was able to facilitate quantification of disease level and its corresponding bioluminescent signal range which can be useful in evaluation of prospective therapies. Recent studies have suggested that the BM microenvironment contributes to the progression of leukemia (16) where cross-talk with stroma favors their localization (17-19). Among many contributing factors, interleukin (IL)-7, produced by stroma cells, has been implicated as playing a significant role in promoting the viability and proliferation of leukemic cells. Expedited progression of leukemia by inducing the upregulation of Bcl-2 and downregulation of $\mathrm{p} 27^{\mathrm{Kip} 1}$ was found to be IL-7-dependent (20). Another study reported that leukemia cell growth preferentially occurs in BM, moreover, leukemic cells can transform normal stromal environment into its favorable malignant microenvironment (21). Therefore, our rationale for IBMT approach to establish leukemia animal model was based on evidence that bone marrow microenvironment plays a significant role in promoting survival and proliferation of leukemic cells (22). 


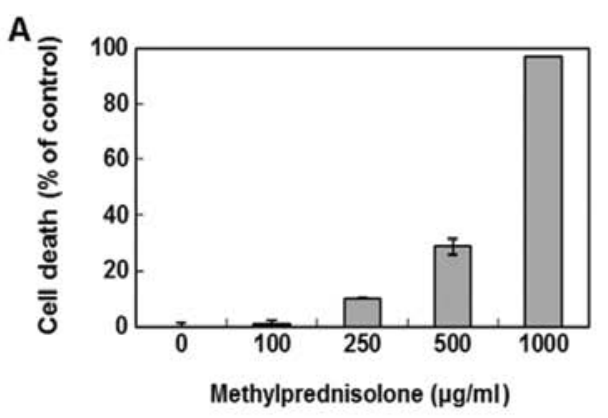

B

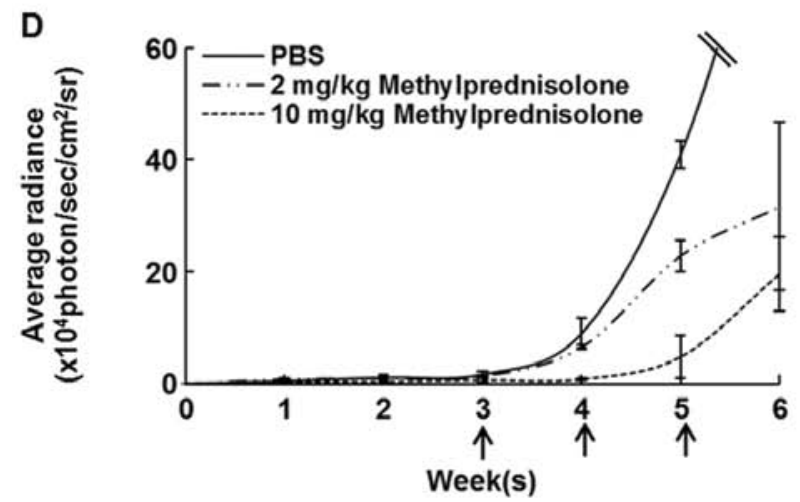

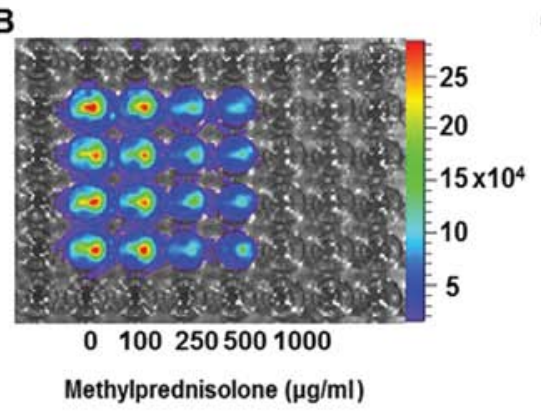

E

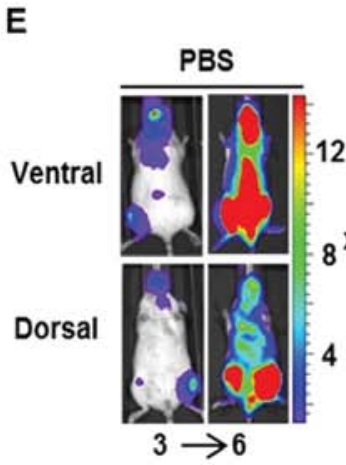

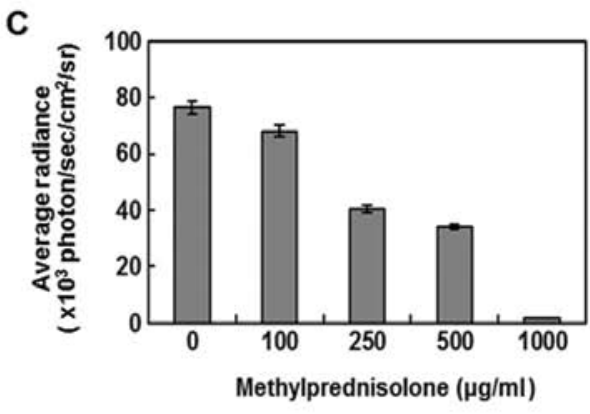

Figure 5. Bioluminescent imaging-based evaluation of sensitivity to methylprednisolone in the IBMT model. (A), In vitro sensitivity of CCRF-CEM/fLuc to methylprednisolone $(0,100,250,500$, or $1000 \mathrm{~g} / \mathrm{ml})$ by Alamarblue assay. (B, C), Bioluminescence of the cell line treated with methylprednisolone at different concentrations. Cell death of CCRF-CEM/fLuc cell line increased and bioluminescence decreased with methylprednisolone concentration. (D), Quantification of bioluminescent CCRF-CEM/fLuc responses over time to weekly intravenous tail-vein treatment of methylprednisolone (100 $\mu 1$ of $2 \mathrm{or} 10 \mathrm{mg} / \mathrm{kg}$ for $3 \mathrm{weeks})$ or PBS (100 $\mu \mathrm{l}$ was given every week for 3 weeks) only. (E), Bioluminescent leukemia responses in control mouse and to 2 and $10 \mathrm{mg} / \mathrm{kg}$ methylprednisolone (MPD) were evaluated by comparing images before treatment at week 3 and after final treatment at week $6(\mathrm{p}<0.01)$.

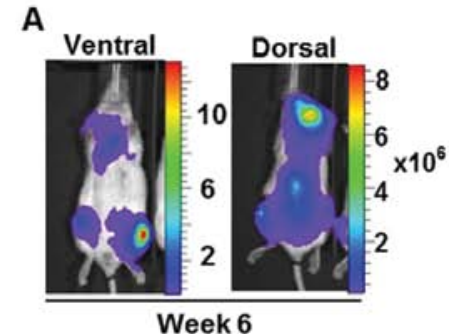

Week 6
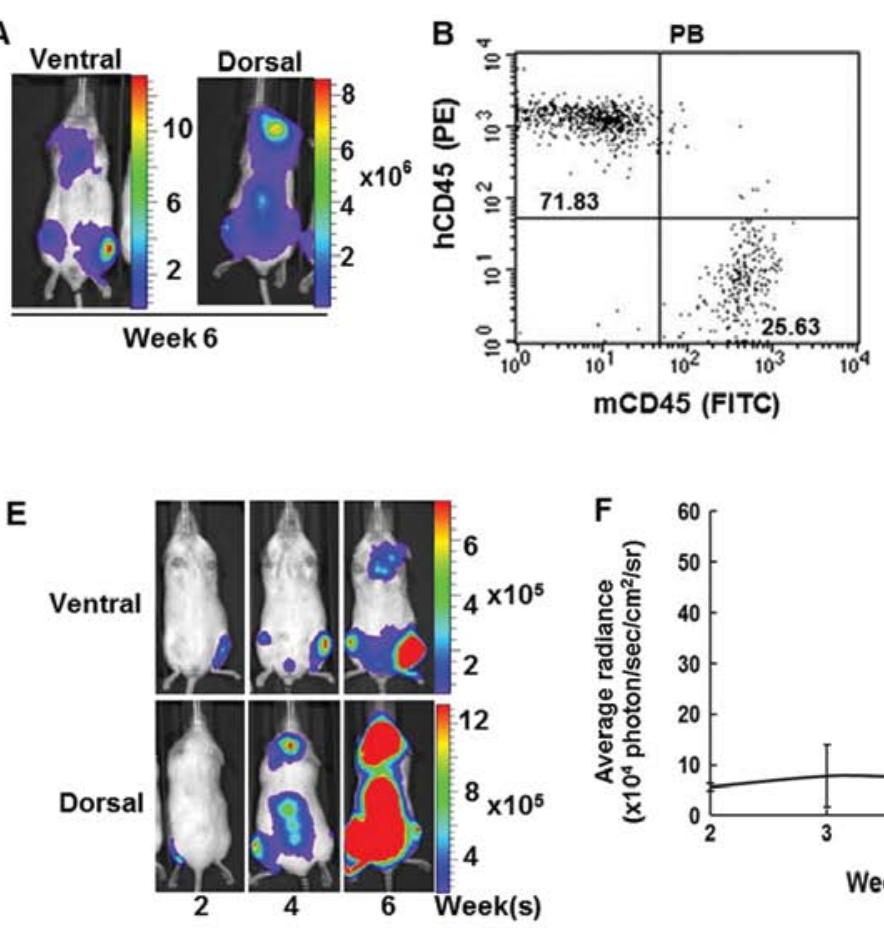
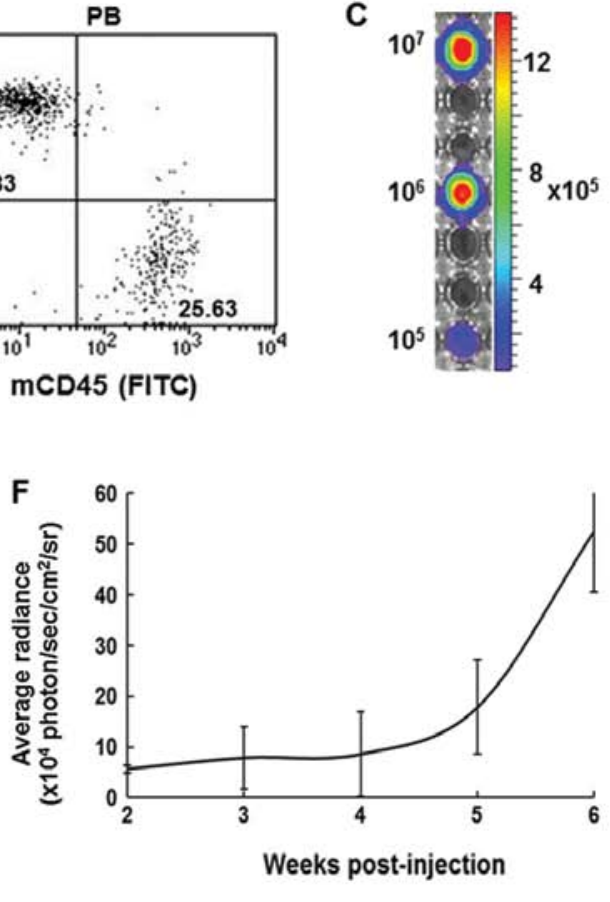
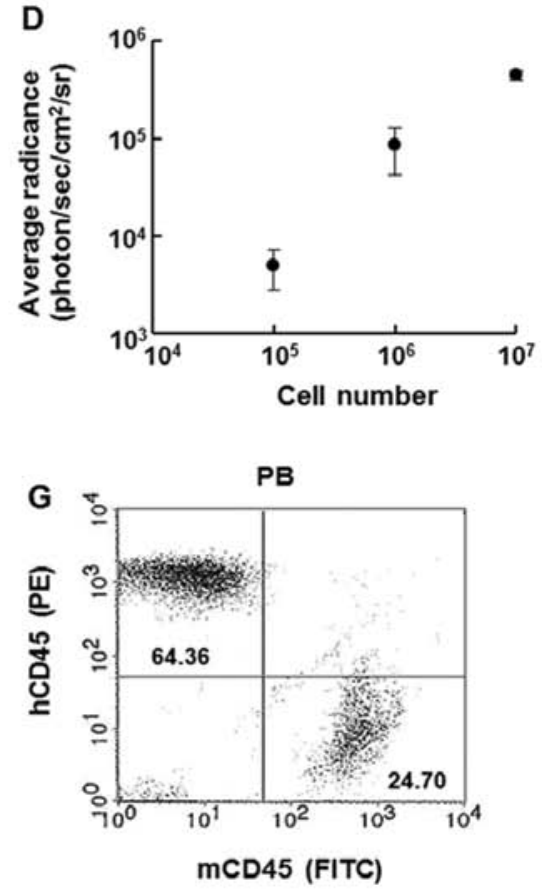

Figure 6. IBMT of bioluminescent CCFR-CEM/fLuc cells into secondary recipient NOD/SCID mice facilitated a reproducible bioluminescent in vivo leukemia model. (A), Bioluminescent image of the first generation of IBMT leukemia model at week 6 , from which the secondary IBMT leukemia model was generated. (B), Flow cytometric characterization of PB of mice at week 6 is shown in (A). Leukemia cells are hCD45+. (C), Imaging of the bioluminescence of serial dilutions $\left(10^{7}, 10^{6}\right.$ and $\left.10^{5}\right)$ of ex vivo expansion-CCRF-CEM/fLuc cells isolated from PB of the first generation of the IBMT leukemia model and (D), represented graphically. (E), Monitoring of the secondary engraftment of bioluminescent leukemia in second generation recipient NOD/SCID over 6 weeks. Bioluminescence images overlaid on photographic images of secondary recipient mice demonstrate that leukemic cells had disseminated evenly. (F), Secondary engraftment of bioluminescent leukemia in second generation recipient NOD/SCID mice $(\mathrm{n}=5)$ presented graphically. (G), Flow cytometric analysis for hCD45 and mCD45 in PB from secondary recipient bioluminescent leukemia in vivo model at week 6 post IBMT. Leukemia cells are hCD45'. 
A growing number of in vivo models for experimental therapeutics relevant to human cancers, such as hematological malignancies, are used in combination with a bioluminescent imaging system to trace leukemia progression in real-time and elucidate underlying biological mechanisms of leukemogenesis (23-27). Furthermore, the combination of bioluminescence and imaging has superseded previous invasive monitoring methods, including serial tail-vein PB sampling, as a more desirable alternative (28). The bioluminescent imaging evaluation is equipped with a powerful technique for cancer cell labeling in which expression of a reporter gene (usually firefly luciferase) provides high detection sensitivity and can be imaged for both spatial and quantitative information (29). Importantly, to use bioluminescent images reliably for preclinical validation of leukemia treatments, in vivo models should incorporate the range of detectable bioluminescent signals that reflects leukemic cell activity in model mice. Leukemia studies using in vivo models have commonly used IV (tail-vein) and IP injections as methods to deliver bioluminescent cells (15,30-33).

In this study, we demonstrated that in vivo bioluminescent monitoring was possible with IBMT of leukemic cells by comparing the bioluminescent images of the three leukemia models over the course of 6 weeks. The comparison revealed that leukemic cells were detected earlier in IBMT model compared to IP and IV models. High intensity bioluminescent signals were visible and detectable in multiple sites of IBMT leukemic model mouse. However, bioluminescent image-based comparison was difficult using images alone because bioluminescent signal ranges for all three models greatly varied and differences between these ranges were too wide to conduct comparison. Especially, in IP and IV models luminescent signal values were significantly lower than IBMT. Therefore, in order to compare the three models, luminescent values displayed as color scale bar must be evaluated along with the images. This color scale bar represents level of intensity of the leukemic cell bioluminescent signals accumulated during $1 \mathrm{~min}$ of imaging. Considering these factors, we have chosen week 5 images of the three leukemia models and adjusted images so that large bioluminescent signal gap between these models can be reduced. High signal regions indicated by red color observed from the IP and IV model images of week 5 have changed to lower signal intensity indicated by blue-violet color whereas high signal regions expanded and intensified in the IBMT model. The comparison of the bioluminescent images and its bioluminescent signal values revealed that leukemic cells in IBMT model were populated systemically and exhibited strong luciferase activity compared to IP and IV models.

In addition, we investigated whether the correlation between bioluminescent signals observed in images and presence of human leukemic cells in the model mice can be established. Although correlation between the signals and level of human leukemic cells were established using bioluminescent images of isolated organs and cells from PB, BM and spleen, we could only found very small portion in IP and IV models whereas large percentage human leukemic cells would be detected in IBMT model. High intensity of bioluminescent signals from the image data could be traced to bioluminescent images of internal organs and significantly higher percentage of hCD $45^{+}$cells were identified in PB (79.06\%) and BM (96.89\%) of IBMT model than of IP and IV models. Bioluminescent signal intensity concentrated in localized region of the IP models often saturated image which made assessment of leukemia burden difficult. These finding suggested that for the purpose of therapeutic response studies, such small number of leukemic cells present in IP and IV models could provide misleading results of in vivo leukemic cell responses to potential therapeutic candidates. In addition, variations between experimental cohorts could arise from IP- and IV-injected leukemic cells becoming lodged in tissue microvasculature, unable to migrate to the proliferation site. These delivery routes may have contributed to delayed progression of leukemia in mice since the engraftment rates of leukemic cells appear to be dependent on the homing potential and time required to reach its microenvironment from the blood $(34,35)$. Another aspect of bioluminescent in vivo model must be considered is that since large bioluminescent signals represent large number of leukemic cells in IBMT model mouse, any changes in these values can be representative data of in vivo leukemic cell responses. We demonstrated that bioluminescent IBMT model can be used to evaluate in vivo responses to antileukemic drugs using well-known drugs such as vincristine and methylprednisolone.

Experiments using primary leukemia cells are integral in studies for elucidating critical molecular targets, drug screening and preclinical validation to establish new therapeutic strategy. Unfortunately, maintaining sufficient numbers of primary leukemia cells ex vivo is inherently limiting because, unlike cell lines, they undergo spontaneous apoptosis in vitro (36-38). Such limited availability of these cells renders experiments reliant on the introduction of the luciferase gene particularly challenging. In 2011, Barrett et al proposed a bioluminescent approach for a non-invasive disease monitoring model system using primary human leukemic cells obtained from in vivo expansion in mice. They demonstrated that it was possible to improve primary cell expansion and detect engraftment rates in immunodeficient mice using in vivo-cultured bioluminescent primary leukemia cells (39). However, their approach in establishing an in vivo model for patient primary leukemia used IV injection, which may have contributed to the finding that greater than $1 \%$ human leukemic cells in PB could only be detected when bioluminescent leukemia burden had reached greater than $1 \times 10^{10}$ photon $/ \mathrm{sec} / \mathrm{sr} / \mathrm{cm}^{2}$ inconsistently. We believe that IBMT could reduce varietal differences and improve sensitivity of preclinical primary leukemia in vivo model to avoid mischaracterization of pharmacodynamics and remedial effects of anti-leukemic drugs.

In conclusion, this study has demonstrated that intramedullary xenograft of leukemia cells into its favored microenvironment, such as the tibia of NOD/SCID mice, can result in consistent establishment of systemic leukemia in an in vivo model. Injection of leukemic cells by other routes, namely IP and IV, provided inconsistent and highly variable leukemia development patterns in NOD/SCID mice with a limited range of bioluminescent signals. Based on these findings, the bioluminescent IBMT in vivo leukemia model could improve current non-invasive longitudinal disease monitoring. It enables the quantitative data range for bioluminescent imaging-based analysis to be extended, allowing more sensitive evaluation of disease burden, more precise quantification of treatment responses. Finally, further adaptation of the IBMT approach to the bioluminescent primary leukemia model could advance developments of novel leukemia patient-tailored therapies. 


\section{Acknowledgements}

This research was supported by Basic Science Research Program through the National Research Foundation of Korea (NRF) funded by the Ministry of Education, Science and Technology (2011-0015112).

\section{References}

1. Seaman ME, Contino G, Bardeesy N and Kelly KA: Molecular imaging agents: impact on diagnosis and therapeutics in oncology. Expert Rev Mol Med 12: e20, 2010.

2. Desar IM, van Herpen CM, van Laarhoven HW, Barentsz JO, Oyen WJ and van der Graaf WT: Beyond RECIST: molecular and functional imaging techniques for evaluation of response to targeted therapy. Cancer Treat Rev 35: 309-321, 2009.

3. Shu ST, Nadella MV, Dirksen WP, et al: A novel bioluminescent mouse model and effective therapy for adult T-cell leukemia/ lymphoma. Cancer Res 67: 11859-11866, 2007.

4. Siegers GM, Felizardo TC, Mathieson AM, Kosaka Y, Wang XH, Medin JA and Keating A: Anti-leukemia activity of in vitroexpanded human gamma delta T cells in a xenogeneic $\mathrm{Ph}^{+}$leukemia model. PLoS One 6: e16700, 2011.

5. Inoue Y, Izawa K, Tojo A, Nomura Y, Sekine R, Oyaizu N and Ohtomo K: Monitoring of disease progression by bioluminescence imaging and magnetic resonance imaging in an animal model of hematologic malignancy. Exp Hematol 35: 407-415, 2007.

6. Bhadri VA, Cowley MJ, Kaplan W, Trahair TN and Lock RB: Evaluation of the NOD/SCID xenograft model for glucocorticoidregulated gene expression in childhood B-cell precursor acute lymphoblastic leukemia. BMC Genomics 12: 565, 2011.

7. Nijmeijer BA, Willemze R and Falkenburg JH: An animal model for human cellular immunotherapy: specific eradication of human acute lymphoblastic leukemia by cytotoxic $\mathrm{T}$ lymphocytes in NOD/scid mice. Blood 100: 654-660, 2002.

8. Fichtner I, Becker M and Baumgart J: Antileukaemic activity of treosulfan in xenografted human acute lymphoblastic leukaemias (ALL). Eur J Cancer 39: 801-807, 2003.

9. Lock RB, Liem N, Farnsworth ML, et al: The nonobese diabetic/ severe combined immunodeficient (NOD/SCID) mouse model of childhood acute lymphoblastic leukemia reveals intrinsic differences in biologic characteristics at diagnosis and relapse. Blood 99: 4100-4108, 2002.

10. Nijmeijer BA, Mollevanger P, van Zelderen-Bhola SL, KluinNelemans HC, Willemze R and Falkenburg JH: Monitoring of engraftment and progression of acute lymphoblastic leukemia in individual NOD/SCID mice. Exp Hematol 29: 322-329, 2001.

11. Inoue Y, Izawa K, Kiryu S, Kobayashi S, Tojo A and Ohtomo K: Bioluminescent evaluation of the therapeutic effects of total body irradiation in a murine hematological malignancy model. Exp Hematol 36: 1634-1641, 2008.

12. Jenkins DE, Oei Y, Hornig YS, et al: Bioluminescent imaging (BLI) to improve and refine traditional murine models of tumor growth and metastasis. Clin Exp Metastasis 20: 733-744, 2003.

13. Sipkins DA, Wei X, Wu JW, et al: In vivo imaging of specialized bone marrow endothelial microdomains for tumour engraftment. Nature 435: 969-973, 2005.

14. Fragoso R, Pereira T, Wu Y, Zhu Z, Cabecadas J and Dias S: VEGFR-1 (FLT-1) activation modulates acute lymphoblastic leukemia localization and survival within the bone marrow, determining the onset of extramedullary disease. Blood 107: 1608-1616, 2006.

15. Bonnet D and Dick JE: Human acute myeloid leukemia is organized as a hierarchy that originates from a primitive hematopoietic cell. Nat Med 3: 730-737, 1997.

16. Ayala F, Dewar R, Kieran M and Kalluri R: Contribution of bone microenvironment to leukemogenesis and leukemia progression. Leukemia 23: 2233-2241, 2009.

17. Zhou J, Mauerer K, Farina L and Gribben JG: The role of the tumor microenvironment in hematological malignancies and implication for therapy. Front Biosci 10: 1581-1596, 2005.

18. Veiga JP, Costa LF, Sallan SE, Nadler LM and Cardoso AA: Leukemia-stimulated bone marrow endothelium promotes leukemia cell survival. Exp Hematol 34: 610-621, 2006.

19. Schmitz M, Breithaupt P, Scheidegger N, et al: Xenografts of highly resistant leukemia recapitulate the clonal composition of the leukemogenic compartment. Blood 118: 1854-1864, 2011.
20. Silva A, Laranjeira AB, Martins LR, et al: IL-7 contributes to the progression of human T-cell acute lymphoblastic leukemias. Cancer Res 71: 4780-4789, 2011.

21. Colmone A, Amorim M, Pontier AL, Wang S, Jablonski E and Sipkins DA: Leukemic cells create bone marrow niches that disrupt the behavior of normal hematopoietic progenitor cells. Science 322: 1861-1865, 2008.

22. Ninomiya M, Abe A, Katsumi A, et al: Homing, proliferation and survival sites of human leukemia cells in vivo in immunodeficient mice. Leukemia 21: 136-142, 2007.

23. Rauch D, Gross S, Harding J, Niewiesk S, Lairmore M, PiwnicaWorms D and Ratner L: Imaging spontaneous tumorigenesis: inflammation precedes development of peripheral NK tumors. Blood 113: 1493-1500, 2009.

24. Inoue Y, Tojo A, Sekine R, et al: In vitro validation of bioluminescent monitoring of disease progression and therapeutic response in leukaemia model animals. Eur J Nucl Med Mol Imaging 33: 557-565, 2006.

25. Lassailly F, Griessinger E and Bonnet D: 'Microenvironmental contaminations' induced by fluorescent lipophilic dyes used for non-invasive in vitro and in vivo cell tracking. Blood 115: 5347-5354, 2010.

26. Tsai HJ, Kobayashi S, Izawa K, et al: Bioimaging analysis of nuclear factor-kappaB activity in Philadelphia chromosomepositive acute lymphoblastic leukemia cells reveals its synergistic upregulation by tumor necrosis factor-alpha-stimulated changes to the microenvironment. Cancer Sci 102: 2014-2021, 2011.

27. Liem NL, Papa RA, Milross CG, et al: Characterization of childhood acute lymphoblastic leukemia xenograft models for the preclinical evaluation of new therapies. Blood 103: 3905-3914, 2004.

28. Zhou B, Ju SG, Ju SW, Xie F and Zhang XG: Establishment of human acute monocytic leukemia model in severe combined immunodeficient (SCID) mice and the analysis of pathological changes. Xi Bao Yu Fen Zi Mian Yi Xue Za Zhi 23: 501-503, 2007.

29. Mezzanotte L, Fazzina R, Michelini E, Tonelli R, Pession A, Branchini $B$ and Roda A: In vivo bioluminescence imaging of murine xenograft cancer models with a red-shifted thermostable luciferase. Mol Imaging Biol 12: 406-414, 2010.

30. Samuels AL, Peeva VK, Papa RA, et al: Validation of a mouse xenograft model system for gene expression analysis of human acute lymphoblastic leukaemia. BMC Genomics 11: 256, 2010.

31. Komeno Y, Kitaura J, Watanabe-Okochi N, et al: AID-induced T-lymphoma or B-leukemia/lymphoma in a mouse BMT model. Leukemia 24: 1018-1024, 2010.

32. Barabe F, Kennedy JA, Hope KJ and Dick JE: Modeling the initiation and progression of human acute leukemia in mice. Science 316: 600-604, 2007.

33. Hoyos V, Savoldo B, Quintarelli C, et al: Engineering CD19specific $\mathrm{T}$ lymphocytes with interleukin-15 and a suicide gene to enhance their anti-lymphoma/leukemia effects and safety. Leukemia 24: 1160-1170, 2010.

34. Messinger Y, Chelstrom L, Gunther R and Uckun FM: Selective homing of human leukemic B-cell precursors to specific lymphohematopoietic microenvironments in SCID mice: a role for the beta 1 integrin family surface adhesion molecules VLA-4 and VLA-5. Leuk Lymphoma 23: 61-69, 1996.

35. Cesano A, O'Connor R, Lange B, Finan J, Rovera G and Santoli D: Homing and progression patterns of childhood acute lymphoblastic leukemias in severe combined immunodeficiency mice. Blood 77: 2463-2474, 1991.

36. Biagi E, Bambacioni F, Gaipa G, Casati C, Golay J, Biondi A and Introna M: Efficient lentiviral transduction of primary human acute myelogenous and lymphoblastic leukemia cells. Haematologica 86: 13-16, 2001.

37. Konopleva M, Konoplev S, Hu W, Zaritskey AY, Afanasiev BV and Andreeff M: Stromal cells prevent apoptosis of AML cells by up-regulation of anti-apoptotic proteins. Leukemia 16: 1713-1724, 2002 .

38. Horisberger MA: A method for prolonged survival of primary cell lines. In Vitro Cell Dev Biol Anim 42: 143-148, 2006.

39. Barrett DM, Seif AE, Carpenito C, et al: Noninvasive bioluminescent imaging of primary patient acute lymphoblastic leukemia: a strategy for preclinical modeling. Blood 118: e112-117, 2011. 